\title{
Publisher's Note: Graphene under the influence of Aharonov-Bohm flux and constant magnetic field
} [Phys. Rev. B 94, 094101 (2016)]

E. A. Stepanov and V. Ch. Zhukovsky

(Received 27 October 2016; published 4 November 2016)

DOI: 10.1103/PhysRevB.94.179902

This paper was published online on 1 September 2016 with an error in Eq. (29).

Equation (29) should read as

$$
\mathcal{L}_{\text {eff }}=-\frac{1}{8 \pi^{2}} \int_{0}^{\infty} \frac{d z}{z} e^{-z m^{2}}\left[(|e| B / z) \operatorname{coth}(|e| z B)-\frac{1}{z^{2}}-\frac{e^{2} B^{2}}{3}\right] .
$$

The equation has been corrected as of 31 October 2016. The equation is incorrect in the printed version of the journal. 